\title{
Teste de respiração espontânea como preditor da Resposta disfuncional ao desmame ventilatório em terapia intensiva
}

Spontaneous breathing test as a predictor of the dysfunctional ventilatory weaning response in intensive care

Prueba de respiración espontánea como predictora de la respuesta disfuncional a la desconexión del ventilador en cuidados intensivos

\section{Priscila Carvalho de SouzaI, Ingrid Régia Lopes Jerônimo"I Carla de Azevedo Vianna ${ }^{\text {III, }}$ Andrea Barroso Benevides ${ }^{\mathrm{IV}}$, Rosimere Ferreira Santana ${ }^{\mathrm{V}}$, Marcos Antônio Gomes Brandão ${ }^{\mathrm{VI}}$}

Resumo: Objetivo: verificar a associação do teste de respiração espontânea com o diagnóstico de enfermagem Resposta disfuncional ao desmame ventilatório (00034) e identificar as medidas de acurácia do teste de respiração espontânea para o diagnóstico de enfermagem. Método: estudo transversal com 42 pacientes em cuidado crítico. $\mathrm{O}$ Teste Exato de Fischer verificou a associação, e as medidas de acurácia foram calculadas com o Medcalc Software Online ${ }^{\circledR}$ Resultados: a associação entre o teste e o diagnóstico foi significativa $(\mathrm{p}=0,0079)$. As medidas de acurácia destacadas foram especificidade $77,42 \%(58,90$ - 90,41), razão de verossimilhança positiva $3,22(1,53$ - 6,79) e odds ratio diagnóstica 9,14 (1,90 - 44,01). Conclusão: o estudo verificou a existência de associação entre o desfecho do teste de respiração espontânea e a Resposta disfuncional ao desmame ventilatório (00034), com significância estatística. Também indicou adequação de três medidas de acurácia para o diagnóstico de enfermagem.

Descritores: Desmame do respirador; Respiração artificial; Testes de função respiratória; Diagnóstico de enfermagem; Tomada de decisões

\footnotetext{
${ }^{\text {I }}$ Enfermeira. Residente em Clínica Médica e Cirúrgica. Universidade Federal do Estado do Rio de Janeiro - UNIRIO. Rio de Janeiro, RJ, Brasil. E-mail: pri.ufrj.micro@gmail.com, ORCID: https://orcid.org/0000-0001-9507-4878.

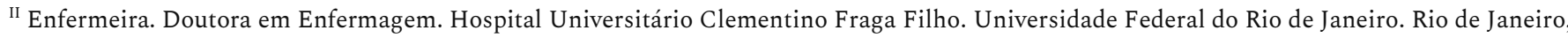
RJ, Brasil. E-mail: ingridregia@gmail.com, ORCID: https://orcid.org/0000-0002-6648-7646.

III Enfermeira. Pós Graduada em Terapia Intensiva e Mestranda em Enfermagem. Escola de Enfermagem Anna Nery. Universidade Federal do Rio de Janeiro. Rio de Janeiro, RJ, Brasil, E-mail: carlinhaavianna@hotmail.com, ORCID: https://orcid.org/0000-0003-4778-7800.

IV Graduanda em Enfermagem. Escola de Enfermagem Anna Nery. Universidade Federal do Rio de Janeiro. Rio de Janeiro, RJ, Brasil. E-mail: abbenevides.enf@gmail.com, ORCID: https://orcid.org/0000-0002-9035-5766.

v Enfermeira. Doutora em Enfermagem. Universidade Federal Fluminense. Niterói, RJ, Brasil. E-mail: rfsantana@id.uff.br, ORCID: https://orcid.org/0000-0002-4593-3715.

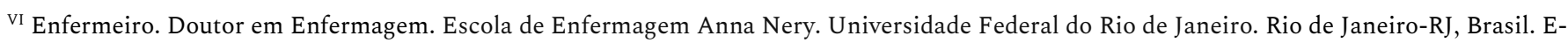
mail: marcosbrandao@ufrj.br, ORCID: https://orcid.org/0000-0002-8368-8343.
} 


\begin{abstract}
Objective: to verify the association of the spontaneous breathing test with the nursing diagnosis of Dysfunctional Ventilatory Weaning Response (00034) and to identify the accuracy measures of the spontaneous breathing test for the nursing diagnosis. Method: a cross-sectional study conducted with 42 patients in critical care. Fischer's exact test verified the association, and the accuracy measures were calculated with Medcalc Software Online ${ }^{\circ}$. Results: the association between the test and the diagnosis was significant $(\mathrm{p}=0.0079)$. The highlighted accuracy measures were $77.42 \%$ specificity $(58.90-90.41)$, positive likelihood ratio of $3.22(1.53-6.79)$, and diagnostic odds ratio of $9.14(1.90-44.01)$. Conclusion: the study verified the existence of an association between the outcome of the spontaneous breathing test and the Dysfunctional Ventilatory Weaning Response (00034), with statistical significance. It also indicated the adequacy of three accuracy measures for the nursing diagnosis.

Descriptors: Ventilator weaning; Respiration, artificial; Respiratory function tests; Nursing diagnosis; Decision making
\end{abstract}

Resumen: Objetivo: verificar la asociación de la prueba de respiración espontánea con el diagnóstico de enfermería de Respuesta disfuncional a la desconexión del ventilador (00034) e identificar las medidas de exactitud de la prueba de respiración espontánea para el diagnóstico de enfermería. Método: estudio transversal realizado con 42 pacientes en cuidados críticos. La prueba exacta de Fischer verificó la asociación, y las medidas de exactitud se calcularon con Medcalc Software Online ${ }^{\bullet}$. Resultados: la asociación entre la prueba y el diagnóstico fue significativa $(\mathrm{p}=0,0079)$. Las medidas de exactitud destacadas fueron las siguientes: especificidad, 77,42\% (58,90 90,41), razón de verosimilitud positiva, 3,22 (1,53 - 6,79) y Odds Ratio diagnóstica, 9,14 (1,90 - 44,01). Conclusión: el estudio verificó la existencia de una asociación entre el resultado de la prueba de respiración espontánea y la Respuesta disfuncional a la desconexión del ventilador (00034), con significancia estadística. También indicó la adecuación de tres medidas de exactitud para el diagnóstico de enfermería.

Descriptores: Desconexión del ventilador; Respiración artificial; Pruebas de función respiratoria; Diagnóstico de enfermería; Toma de decisiones

\title{
Introdução
}

A ventilação mecânica $(\mathrm{VM})$ é um recurso essencial para a manutenção da vida de pacientes gravemente enfermos. Apesar de ser uma terapêutica necessária, pode induzir complicações capazes de aumentar a morbimortalidade dos mesmos, resultando em alto custo institucional, e produzir desconforto, sendo prioritário o restabelecimento da ventilação espontânea o mais breve possível. ${ }^{1-2} \mathrm{O}$ processo de desmame ventilatório marca a transição da respiração artificial com suporte mecânico para a respiração espontânea e evolui, usualmente, para a extubação com a remoção do tubo endotraqueal. ${ }^{3}$

Pelas Diretrizes Brasileiras de Ventilação Mecânica, o sucesso do desmame ocorre quando o paciente tolera o Teste de Respiração Espontânea (TRE) ainda conectado ao ventilador 
3 | Souza PC, Jerônimo IR, Vianna CA, Benevides AB, Santana RF, Brandão MAG

mecânico. ${ }^{4}$ Por conta disso, o mencionado teste tem assumido um papel importante, levando em conta que é o melhor método para avaliar a capacidade da extubação. ${ }^{5-6}$ Ele consiste na interrupção da VM e na manutenção do paciente em ventilação espontânea sem a retirada da via aérea artificial, podendo durar de 30 a 120 minutos. $^{5}$

Apesar disso, o desmame e a extubação seguem como faces de um fenômeno complexo no qual tomadas de decisão inapropriadas podem aumentar o trabalho de respiração com falência respiratória e cardíaca ou produzir uma disfunção diafragmática induzida pelo ventilador em processos mais prolongados. ${ }^{6-7} \mathrm{~A}$ avaliação incorreta da prontidão para extubação pode levar à necessidade de reintubação. Uma revisão sistemática com metanálise evidenciou que a reintubação intensifica o risco, com odds ratio de 7,57, para a pneumonia associada à ventilação e de 3,33 para a mortalidade hospitalar. ${ }^{8}$

Um estudo na enfermagem que acompanhou o desmame durante a realização de TRE por 120 minutos verificou que a taxa de insucesso foi de $47,3 \%$ do total de casos acompanhados. ${ }^{9}$ Portanto, avaliar e estimar o sucesso do desmame ventilatório é um desafio, como o monitoramento das respostas ao processo.

Do ponto de vista da avaliação diagnóstica de enfermagem, o desmame pode desencadear no paciente duas respostas humanas diretamente relacionadas a ele: a resposta normal ou a resposta disfuncional. ${ }^{10}$ A Resposta disfuncional ao desmame ventilatório (00034) foi incorporada à classificação diagnóstica da North American Nursing Diagnosis Association (NANDA) em 1992 e hoje é definida na NANDA-I Taxonomy como "a incapacidade de ajustar-se a níveis diminuídos de suporte ventilatório mecânico, que interrompe e prolonga o processo de desmame”. 11:235

Os diagnósticos são julgamentos sobre respostas humanas, ou seja, comportamentos biológicos, sociais, psíquicos e até mesmo espirituais do indivíduo, família e/ou coletividade. Por sua complexidade, são de improvável identificação somente pelo emprego de dispositivos e equipamentos. $^{12}$ Assim, cabe ao enfermeiro a observação do paciente, a centralidade e 
Teste de respiração espontânea como preditor da Resposta disfuncional ao... | 4

assertividade na detecção de respostas anormais, selecionando intervenções adequadas ao paciente. ${ }^{1}$ Entretanto, a identificação acurada de uma resposta humana pode ser difícil de realizar, e muitas vezes, depende do treinamento do diagnosticistas. ${ }^{13}$

Estudos sobre indicadores clínicos ou características definidoras apresentam medidas para estimativa da Resposta disfuncional ao desmame ventilatório (00034). ${ }^{8,14}$ A literatura também possui estudos que apontam o TRE como bom preditor do sucesso ou insucesso do desmame.,15-16

Por outro lado, pouco se pode afirmar sobre as relações existentes entre o diagnóstico de enfermagem e a predição do sucesso do processo de desmame. Também, a relação do TRE com o diagnóstico de enfermagem Resposta disfuncional ao desmame ventilatório (00034) segue como uma questão de pesquisa a ser investigada para fornecer novas informações sobre a inferência diagnóstica de enfermeiros.

Os objetivos do estudo foram verificar a associação do teste de respiração espontânea com o diagnóstico de enfermagem Resposta disfuncional ao desmame ventilatório (00034) e identificar as medidas de acurácia do teste de respiração espontânea para o diagnóstico de enfermagem.

\section{Método}

Estudo transversal desenvolvido nas unidades de terapia intensiva (UTI) geral e cardiológica de um hospital de ensino, público e federal do sudeste do Brasil. A UTI geral composta por 20 leitos e a UTI cardíaca, por 6 leitos, representando uma das unidades de referência da região. Foram participantes do estudo os pacientes internados com comorbidades clínicas e/ou cirúrgicas que se encontraram mecanicamente ventilados por meio de intubação orotraqueal e acoplamento à prótese ventilatória por um período superior a 24 horas.

Para inclusão, os parâmetros hemodinâmicos dos participantes deveriam estar estáveis hemodinamicamente e atenderem aos demais critérios de avaliação de prontidão para o desmame e a consequente recomendação para o TRE. ${ }^{3}$ Foram excluídos do estudo pacientes 
5 | Souza PC, Jerônimo IR, Vianna CA, Benevides AB, Santana RF, Brandão MAG

intubados portadores de lesões raquimedulares altas, miastenia gravis ou outras desordens neurológicas que interferissem diretamente na capacidade de gerar impulso ventilatório próprio. Aplicando-se os critérios de inclusão foram selecionados 46 pacientes elegíveis e submetidos ao desmame. Entretanto, houve perda de quatro pacientes pela decisão da equipe multiprofissional por realização de traqueostomia, restando 42 para compor a análise do estudo.

No estudo, a falha ou sucesso do TRE referem-se ao teste curto, ou seja, o realizado por 30 minutos. $^{17}$ Sua realização precedeu a extubação do paciente, incorporando avaliação criteriosa da tolerância à respiração espontânea. ${ }^{3}$ Foram parâmetros ventilatórios para o teste: ventilação espontânea do paciente conectado à prótese, com fonte enriquecida de oxigênio em FiO2 de até 40\%, em modalidade de pressão de suporte (PSV) de até $7 \mathrm{cmH}_{2} \mathrm{O}$, e com Pressão Expiratória Final Positiva (PEEP) de $5 \mathrm{cmH}_{2} \mathrm{O}$ em até 120 minutos. ${ }^{18} \mathrm{O}$ desfecho de falha ou sucesso no teste foi julgado por equipe multiprofissional com aplicação dos seguintes critérios: manutenção do padrão respiratório, troca gasosa, estabilidade hemodinâmica, conforto; e ausência de sinais de frequência respiratória $>35$ irpm, saturação arterial de $\mathrm{O}_{2}<90 \%$, frequência cardíaca $>140$ bpm, pressão arterial sistólica $>180 \mathrm{mmHg}$ ou $<90 \mathrm{mmHg}$; sinais e sintomas de agitação, sudorese, alteração do nível de consciência. ${ }^{4}$

Foram dados obtidos do monitoramento multiparamétrico: eletrocardiografia, oximetria de pulso, pressão arterial invasiva ou não invasiva e frequência respiratória. Dados gasométricos do sangue arterial e venoso foram coletados do prontuário. Todos os parâmetros mencionados foram antes do TRE em circunstância na qual o estado do paciente fosse o mais próximo de uma condição basal, de bem-estar e conforto, e obtidos do prontuário eletrônico após a decisão diagnóstica. Posteriormente, a oximetria de pulso, pressões arteriais e frequência respiratória foram novamente avaliadas à beira do leito e anotadas durante o teste, sendo usadas como critério de avaliação de características definidoras. 
Teste de respiração espontânea como preditor da Resposta disfuncional ao... | 6

As características definidoras do diagnóstico de Resposta disfuncional ao desmame ventilatório (00034) foram coletadas durante 120 minutos, selecionadas e documentadas em

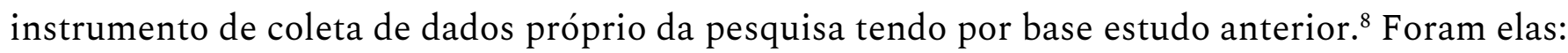
expressão facial de medo; desconforto respiratório; inquietação; aumento da frequência cardíaca menor do que $20 \mathrm{bpm}$ da linha de base; aumento da pressão arterial menor do que $20 \mathrm{mmHg}$ da linha de base; sensação de calor questionada diretamente ao paciente e confirmada com linguagem não verbal; agitação; ruídos adventícios respiratórios; entrada de ar diminuída à ausculta; aumento da concentração respiratória; uso mínimo da musculatura acessória respiratória; aumento da pressão arterial $>20 \mathrm{mmHg}$ respiração abdominal paradoxal; apreensão; aumento moderado da frequência respiratória acima dos valores basais menor do que 5irpm da linha de base; aumento da frequência cardíaca $>20 \mathrm{bpm}$; aumento da frequência respiratória de forma significativa em relação aos parâmetros basais (>5irpm); respiração superficial; medo de mau funcionamento do aparelho; capacidade prejudicada de responder às orientações, sendo compatíveis com a situação de teste para desmame; foco exagerado nas atividades, manifestada no direcionamento excessivo da atenção do paciente para as tarefas realizadas pelos profissional quando do cuidado na assistência ventilatória; uso importante da musculatura acessória respiratória; diaforese; respiração gasping; deterioração dos gases sanguíneos arteriais; capacidade prejudicada em cooperar, sendo compatíveis com a situação de teste para desmame; cor da pele anormal; diminuição do nível de consciência; fadiga.

$\mathrm{Na}$ coleta dos dados, a pesquisadora foi acompanhada por outros dois auxiliares de pesquisa que também realizaram o exame físico do paciente após receberem treinamento teórico e prático para avaliação do diagnóstico de enfermagem que incluiu as definições conceituais e operacionais das características definidoras e a revisão das técnicas de avaliação semiológica do diagnóstico de enfermagem. A inferência diagnóstica de enfermagem cabia à pesquisadora. Posteriormente, a inferência era discutida com a enfermeira de cuidados diretos 
designada ao paciente para obtenção de consenso. Nessa sessão, as informações adicionais trazidas pela enfermeira eventualmente reconfiguravam a inferência inicial.

Em seguida, isoladamente, os dados dos pacientes com as características definidoras foram reavaliados por dois pesquisadores com experiência no diagnóstico de enfermagem, de forma que inferiram sobre a presença ou ausência da Resposta disfuncional ao desmame ventilatório (00034). Comparadas as inferências de campo com a do painel de avaliadores, um debate entre os pesquisadores principais foi realizado para as situações sem concordância até a obtenção de consenso no painel de avaliadores. ${ }^{12}$ A coleta de dados ocorreu de março de 2016 a março 2017.

Os resultados da avaliação do TRE e do julgamento sobre a Resposta disfuncional ao desmame ventilatório (00034) foram organizados em tabela de contingência. Na verificação da associação aplicou-se o Teste exato de Fisher, adotando-se a significância para um valor p<0,05. Para as medidas de acurácia do TRE como estimativa do diagnóstico de enfermagem foram usadas: sensibilidade, especificidade, razão de verossimilhança positiva, razão de verossimilhança negativa, acurácia e odds ratio diagnóstica, com ponto de corte em $70 \%$. A prevalência e valores no intervalo de confiança de $95 \%$ do diagnóstico de enfermagem também foram calculados. Todas as medidas foram obtidas com apoio do Medcalc Software Online ${ }^{\varpi}$ A redação do artigo foi orientada, no que foi apropriado, pelo Reporting Diagnostic Accuracy Studies Guideline (STARD). ${ }^{19}$

A pesquisa da qual deriva o estudo obteve aprovação de Comitê de Ética em Pesquisa com parecer de número 2.021.191 de 10 de março de 2016 e emenda para ampliação de período de coleta de dados no parecer de número 2.040.572 de 02 de maio de 2017. O estudo encontra-se em acordo com os preceitos éticos preconizado pela Portaria 466/2012, do Conselho Nacional Saúde do Brasil.

\section{Resultados}


Teste de respiração espontânea como preditor da Resposta disfuncional ao... $\mid 8$

O fluxo de participantes do estudo é representado na Figura 1, seguindo as recomendações do STARD Guideline.

Figura 1 - Fluxo de participantes do estudo, Rio de Janeiro - RJ, 2020

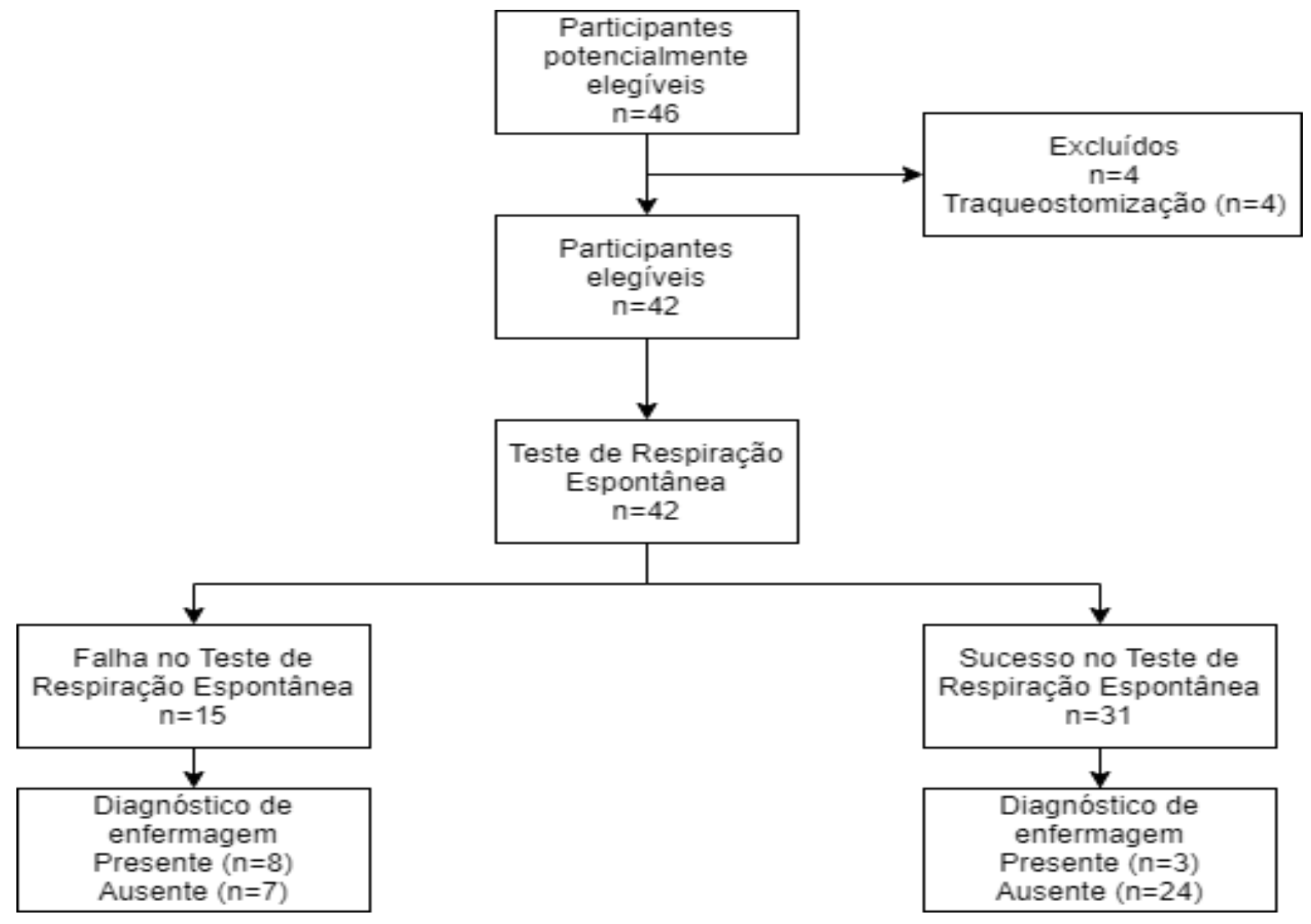

Fonte: Autores, 2020

As características clínicas e sociodemográficas foram: idade entre 18 e 79 anos (média=57,3 anos, desvio padrão de 13,8 anos), com $58,7 \%$ de participantes do sexo feminino. Quanto às causas mais comuns de admissão nas unidades de terapia intensiva, destacaram-se as causas clínicas com $56,5 \%$, seguidas das causas cirúrgicas com $32,6 \%$, e houve ainda $10,9 \%$ dos pacientes com causas coronarianas e cardíacas. Em relação ao tempo de ventilação mecânica invasiva, o intervalo foi de 2 a 21 dias, com mediana de 7,5 dias, média de 8,4 dias e desvio padrão de 4,9 dias. 
9 | Souza PC, Jerônimo IR, Vianna CA, Benevides AB, Santana RF, Brandão MAG

Os dados obtidos no estudo contidos na Tabela 1 indicam uma prevalência do diagnóstico de enfermagem de $26,19 \%$ e de $35,71 \%$ de falha do TRE. A associação entre o mencionado teste e a Resposta disfuncional ao desmame ventilatório (00034) foi verificada pelo valor de significância estatística $(\mathrm{p}=0,0079)$.

Tabela 1 - Associação entre o desfecho no teste de respiração espontânea (TRE) e a Resposta disfuncional ao desmame ventilatório (00034). Rio de Janeiro, Brasil.

\begin{tabular}{lccccc}
\hline Desfecho & \multicolumn{2}{c}{ Resposta disfuncional ao desmame } & $\mathbf{n}$ & $\mathbf{\%}$ & p-valor $^{*}$ \\
& ventilatório & & & \\
\hline Desfecho do TRE & Sim & Não & & & \\
Falha & 8 & 7 & 15 & 35,7 & 0,0079 \\
Sucesso & 3 & 24 & 27 & 64,3 & \\
Total & 11 & 31 & 42 & 100 & \\
\hline
\end{tabular}

Fonte: Dados da pesquisa, 2017.

Legenda: TRE= teste de respiração espontânea. *Teste Exato de Fisher.

A Tabela 2 indica que os valores de sensibilidade $(72,73 \%)$, especificidade $(77,42 \%)$ e acurácia (76,19\%) superaram o ponto de corte estabelecido no estudo. As razões de verossimilhança positiva de 3,22 (IC: 1,53 - 6,79) e a odds ratio diagnóstica (ORD) de 9,14 (IC: 1,89 - 44,01) também são resultados a serem destacados, tanto pelo significado de seus valores absolutos, quanto pela variação calculada nos intervalos de confiança.

Tabela 2 - Medidas de acurácia diagnóstica do teste de respiração espontânea para estimar a ocorrência do diagnóstico de enfermagem. Rio de Janeiro, Brasil.

\begin{tabular}{lc}
\hline Medida de acurácia & Valor $(\mathbf{I C}$ 95\%) \\
\hline Sensibilidade & $72,73(39,03-93,98)$ \\
Especificidade & $77,42(58,90-90,41)$ \\
Razão de Verossimilhança Positiva & $3,22(1,53-6,79)$ \\
Razão de Verossimilhança Negativa & $0,35(0,13-0,94)$ \\
Acurácia & $76,19(60,55-87,95)$ \\
Odds ratio diagnóstica & $9,14(1,89-44,01)$ \\
Prevalência do diagnóstico & $26,19(13,86-42,04)$ \\
\hline
\end{tabular}

Fonte: Dados da pesquisa, 2017. 
Teste de respiração espontânea como preditor da Resposta disfuncional ao... | 10

\section{Discussão}

A associação verificada entre o TRE e a resposta humana expressa no diagnóstico de enfermagem Resposta disfuncional ao desmame ventilatório (00034) é um resultado original e com potencial de ser explorado por enfermeiros intensivistas, incentivando a participação dos mesmos na decisão pela extubação, tendo por base evidências de que o seu diagnóstico pode ser somado aos demais critérios clínicos na estimativa da evolução do indivíduo para saída da assistência ventilatória. Talvez a correlação investigada entre o julgamento de indícios de prontidão para extubação, por meio de um teste rotineiro, com o julgamento diagnóstico de enfermagem possa ser um fator para impulsionar a inferência diagnóstica do enfermeiro.

O estudo verificou taxa de fracasso no TRE inferior a obtida em outro estudo que indicou $47,3 \%$ de falhas, também realizado com adultos internados. ${ }^{9} \mathrm{O}$ insucesso inicial do teste tem sido reportado na literatura com intervalos entre $21 \%$ até $32 \%$, e ao final de todo o processo de desmame, após 48 horas, entre $25,5 \%$ e $42,4 \% .{ }^{16}$

Estudo de validação da Resposta disfuncional ao desmame ventilatório (00034) com adultos internados em Unidades de Terapia Intensiva de Belo Horizonte verificou que, dos 93 pacientes que compuseram a amostra, 41 (44,1\%) apresentaram o diagnóstico, o que pelos critérios adotados naquele estudo, correspondeu à falha na extubação. ${ }^{14}$ Os achados são próximos aos obtidos nesta pesquisa, mas sem usar o TRE como preditor.

Múltiplas variáveis, podem predizer o fracasso do desmame ventilatório, dentre elas: padrão de sono atípico e disfunção do diafragma; força da tosse; idade; e frequência respiratória. ${ }^{3,7,20}$ Para a extubação outros preditores reportados são: secreções excessivas; pressão parcial de gás carbônico $(\mathrm{PaCO} 2)>45 \mathrm{mmHg}$ ); duração da ventilação mecânica $>72 \mathrm{~h}$, problemas nas vias aéreas superiores; e falhas prévias em tentativa de desmame. ${ }^{16}$ Esses preditores para o desfecho do desmame e extubação são coincidentes com características definidoras e fatores relacionados da Resposta disfuncional ao desmame ventilatório (00034). Dentre as 
11 | Souza PC, Jerônimo IR, Vianna CA, Benevides AB, Santana RF, Brandão MAG

características definidoras os aumentos leves, moderados e significativos da frequência respiratória em relação aos parâmetros basais podem ser outra conceitualização para as alterações na frequência respiratória, e os fatores relacionados alteração no padrão de sono e desobstrução ineficaz de vias aéreas como expressões do padrão de sono atípico e da força (diminuída) da tosse. ${ }^{11}$

Diferentes critérios para definir a Resposta disfuncional ao desmame ventilatório (00034) podem modificar a interpretação dos achados. Por conta disso, é relevante recortar claramente os estágios do desmame e da extubação e considerar as diferentes definições operacionais entre estudos, para só então estabelecer comparações. Sinais e sintomas preditores parecem diferir entre o desmame durante o TRE e durante o período da extubação, ou seja, nas 48 horas seguintes. Como exemplo, força da tosse tem sido associada apenas à predição da extubação e não ao desmame, ${ }^{3}$ o que deve ser considerado ao se avaliar a desobstrução ineficaz de vias aéreas como o fator relacionado para o diagnóstico de enfermagem.

Considerando os valores medidos e as estimativas dos intervalos de confiança, as medidas mais acuradas para estimar a relação entre o resultado do teste e o diagnóstico de enfermagem foram a especificidade, a razão de verossimilhança positiva e a ORD. A especificidade pode indicar ao enfermeiro a maior proporção da ausência da resposta disfuncional nos pacientes que tiveram sucesso no teste.

A razão de verossimilhança positiva informa a probabilidade de uma falha no TRE ocorrer em pacientes com a Resposta disfuncional ao desmame ventilatório dividida pela probabilidade de uma falha em pacientes sem o diagnóstico. A razão de verossimilhança negativa informa a probabilidade de sucesso no teste em pacientes com o diagnóstico dividida pela probabilidade do sucesso em pacientes sem o diagnóstico de enfermagem. O resultado superior a 1,0 mesmo no intervalo de confiança indica a validade do TRE para corroborar a presença da Resposta disfuncional ao desmame ventilatório. Por fim, o valor da ORD maior do 
Teste de respiração espontânea como preditor da Resposta disfuncional ao... | 12

que 1,0 no intervalo de confiança indicou maior a chance de falha no teste de respiração espontânea indicar a Resposta disfuncional ao desmame ventilatório (00034).

A combinação do conjunto de medidas de acurácia indica a importância que o TRE pode representar para o diagnóstico de enfermagem. Entretanto, essa resposta disfuncional é um fenômeno complexo que tende a requerer o monitoramento de outras variáveis. Por exemplo, o aumento da frequência respiratória parece ser um indicador relevante para acompanhar o processo de desmame, sendo seus valores de sensibilidade boas estimativas para a diagnóstico de enfermagem e para o fracasso do desmame, bem como pode ser considerada para o cálculo do índice f/Vt (frequência por volume). ${ }^{14,21-23} \mathrm{O}$ alerta ao se usar tais evidências é considerar que os mencionados estudos usaram diferentes definições operacionais para caracterizar o aumento da frequência respiratória, o que exige cuidadosa interpretação para comparações.

Os achados indicam que informações importantes podem ser extraídas da evolução e desfecho do TRE. O sucesso do Teste com ausência do diagnóstico pode antecipar que o processo de extubação poderá cursar com maior conforto para o paciente. Por outro lado, estudos tem demonstrado que efeitos de sobrecarga dos músculos respiratórios, quando ocorrem, tendem a ocorrer no início de um TRE. ${ }^{5,24-25}$ Modelos de regressão logística já são usados para lidar com características definidoras ${ }^{14} \mathrm{e}$, futuramente, podem ser usados para incorporar o desfecho no TRE entre as variáveis consideradas, potencializando a inferência diagnóstica de enfermagem e aproximando a prática multidisciplinar.

Estudos tem sustentado a importância de considerar a existência de um padrão temporal na avaliação das características definidoras da Resposta disfuncional ao desmame ventilatório. $^{14,23}$ Ambos estudos verificaram o aumento da frequência respiratória como evidência do diagnóstico nos primeiros 30 minutos de avaliação, indicando uma prevalência de $46,3 \%$ para a taquipneia ${ }^{14}$ e aumento leve a moderado da frequência respiratória surgindo por volta dos 39-40 minutos de início de um TRE de 120 minutos. ${ }^{23}$ No intervalo seguinte de 60 
minutos a ocorrência mais frequente foi de queda da saturação de oxigênio. ${ }^{14}$ No intervalo de 90 e de 120 minutos os estudos indicaram a ocorrência de sinais mais graves como taquipneia, batimento de aletas nasais, uso de musculatura acessória, alteração no nível de consciência, aumento da pressão sanguínea em relação aos parâmetros basais $(>20 \mathrm{mmHg})$ e sensação expressa de necessidade de oxigênio aumentada. ${ }^{14-18}$

Demonstrou-se o valor inferencial do TRE, rotineiramente aplicado pela equipe multiprofissional para verificar a prontidão do paciente para a extubação, como estimativa diagnóstica de enfermagem. O reconhecimento dessa importância inferencial tende a pontuar o papel do raciocínio clínico de enfermeiros na colaboração com médicos e fisioterapeutas respiratórios na avaliação e tomada de decisão terapêutica, o que em última instância pode facilitar a coordenação de esforços multiprofissionais.

\section{Conclusão}

O estudo verificou a existência de associação entre o desfecho do TRE e a Resposta disfuncional ao desmame ventilatório (00034), com significância estatística. Também indicou adequação de medidas de acurácia diagnóstica de enfermagem, destacando-se a especificidade, a razão de verossimilhança negativa e a odds ratio diagnóstica.

Os resultados demonstraram que o desfecho do TRE pode servir especialmente para os enfermeiros planejarem sua avaliação, sabendo que pacientes que tenham experimentado falha no teste possuem uma chance aumentada de apresentar o diagnóstico. Por outro lado, podem combinar o teste a outras características definidoras acuradas para ampliar a eficiência de seu julgamento clínico.

Apesar da coleta de dados ter compreendido 12 meses, dificuldades estruturais experimentadas pela instituição pesquisada durante esse período não permitiram que fosse obtida uma amostra mais abrangente, o que implicou em intervalos de confiança alargados para 
Teste de respiração espontânea como preditor da Resposta disfuncional ao... | 14

as medidas de acurácia, limitando sobretudo a interpretação dos valores de sensibilidade, valor preditivo negativo e acurácia.

A consequência natural para a translação do conhecimento trazido pelo estudo é a redefinição de papéis e o estabelecimento de uma proporção de recursos humanos que permita ao enfermeiro desenvolver sua relevante atuação na identificação da Resposta disfuncional ao desmame ventilatório (00034), com objetivo de minimizar as respostas desagradáveis experimentadas pelo paciente durante a saída da ventilação mecânica. Entende-se que novas possibilidades de prática colaborativa são trazidas pela pesquisa ao indicar evidências que relacionam o julgamento clínico de enfermagem com um teste multiprofissional de tomada de decisão sobre a interrupção da ventilação, abrindo potencial avanço do campo saúde para a redução de complicações biomédicas e redução da experiência para o paciente de respostas humanas desagradáveis.

\section{Referências}

1. Loss SH, Oliveira RP, Maccari JG, Savi A, Boniatti MM, Hetzel MP, et al. The reality of patients requiring prolonged mechanical ventilation: a multicenter study. Rev Bras Ter Intensiva. 2015;27(1):2635. doi: 10.5935/0103-507X.20150006

2. Girard TD, Alhazzani W, Kress JP, Ouellette DR, Schmidt GA, Truwit JD, et al. An official american thoracic society/american college of chest physicians clinical practice guideline: liberation from mechanical ventilation in critically ill adults. Rehabilitation protocols, ventilator liberation protocols, and cuff leak tests. Am J Respir Crit Care Med. 2017;195(1):120-33. doi: 10.1164/rccm.201610-2075ST

3. Baptistella AR, Sarmento FJ, Silva KR, Baptistella SF, Taglietti M, Zuquello RÁ, et al. Predictive factors of weaning from mechanical ventilation and extubation outcome: a systematic review. J Crit Care. 2018;48:56-62. doi: 10.1016/j.jcrc.2018.08.023

4. Barbas CSV, Ísola AM, Farias AMC, Cavalcanti AB, Gama AMC, Duarte ACM, et al. Brazilian recommendations of mechanical ventilation 2013. Part I. Rev Bras Ter Intensiva. 2014;26(2):89-121. doi:10.5935/0103-507X.20140017

5. Zein H, Baratloo A, Negida A, Safari S. Ventilator weaning and spontaneous breathing trials: an educational review. Emerg (Tehran) [Internet]. 2016 Spring [cited 2019 Oct 22];4(2):65-71. Available from: https://pubmed.ncbi.nlm.nih.gov/27274515/ 
6. Rose L. Strategies for weaning from mechanical ventilation: a state of the art review. Intensive Crit Care Nurs. 2015;31(4):189-95. doi: 10.1016/j.iccn.2015.07.003

7. Medrinal C, Prieur G, Frenoy E, Robledo Quesada A, Poncet A, Bonnevie T, et al. Respiratory weakness after mechanical ventilation is associated with one-year mortality - a prospective study. Crit Care. 2016;20(1):231. doi: 10.1186/s13054-016-1418-y

8. Gao F, Yang LH, He HR, Ma XC, Lu J, Zhai YJ, et al. The effect of reintubation on ventilatorassociated pneumonia and mortality among mechanically ventilated patients with intubation: a systematic review and meta-analysis. Heart Lung. 2016;45(4):363-71. doi: 10.1016/j.hrtlng.2016.04.006

9. Brandão MAG, Cerqueira FA, Matos LN, Campos JF, Peixoto MAP, Primo CC. Defining characteristics of the dysfunctional ventilatory weaning response as indicators of accuracy of ventilatory weaning. Rev Bras Enferm. 2014;67(5):737-43. doi: 10.1590/0034-7167.2014670510

10. Mary A. Post Hoc analysis of cardiopulmonary indicators trends among post-operative coronary artery bypass graft patients with normal ventilator weaning response and dysfunctional ventilator weaning response. J Cardiovasc Surg [Internet]. 2013 [cited 2019 Oct 22];1(2):25. Available from: https://pdfs.semanticscholar.org/81c4/3e0ee3a5ec90ab9a64ecef079ce9134ac30e.pdf

11. Herdman H, Kamitsuru S. Diagnósticos de Enfermagem da NANDA-I: Definições e Classificação 2018/2020. 11를 ed. Porto Alegre: Artmed; 2018.

12. Martins V, Silva DA. Métodos avançados de validação de diagnósticos de enfermagem. Porto Alegre: Artmed/Panamericana; 2016. 3 vol. PRONANDA Programa atualização em diagnósticos de enfermagem; p. 9-51.

13. Lopes MVO, Silva VM, Araujo TL. Methods for establishing the accuracy of clinical indicators in predicting nursing diagnoses. Int J Nurs Knowl. 2012;23(3):134-9. doi: 10.1111/j.2047-3095.2012.01213.x

14. Silva LCR. Validação clínica do diagnóstico de enfermagem "Resposta disfuncional ao desmame ventilatório” em pacientes adultos internados em Unidade de Terapia Intensiva [dissertação]. Belo Horizonte: Universidade Federal de Minas Gerais; 2017 [acesso em 2019 nov 10]. 202f. Disponível em: http://hdl.handle.net/1843/ANDO-AN4GMU

15. Liang G, Liu T, Zeng Y, Shi Y, Yang W, Yang Y, et al. Characteristics of subjects who failed a 120minute spontaneous breathing trial. Respir Care. 2018;63(4):388-94. doi: 10.4187/respcare.05820

16. Ouellette DR, Patel S, Girard TD, Morris PE, Schmidt GA, Truwit JD, et al. Liberation from mechanical ventilation in critically ill adults: an official American College of Chest Physicians/American Thoracic Society Clinical practice guideline. Chest. 2017;151(1):166-80. doi: 10.1016/j.chest.2016.10.036

17. Figueroa-Casas JB, Connery SM, Montoya R. Changes in breathing variables during a 30-minute spontaneous breathing trial. Respir Care. 2015;60(2):155-61. doi: 10.4187/respcare.03385 
Teste de respiração espontânea como preditor da Resposta disfuncional ao... | 16

18. Nanchal RS, Truwit JD. Advancing weaning science: shorter or longer duration of SBT? Illuminating the road ahead. Respir Care. 2018;63(4):493-4. doi: 10.4187/respcare.06066

19. Bossuyt PM, Reitsma JB, Bruns DE, Gatsonis CA, Glasziou PP, Irwig L, et al. STARD 2015: an updated list of essential items for reporting diagnostic accuracy studies. BMJ. 2015;351:h5527. doi: 10.1136/bmj.h5527

20. Thille AW, Reynaud F, Marie D, Barrau S, Rousseau L, Rault C, et al. Impact of sleep alterations on weaning duration in mechanically ventilated patients: a prospective study. Eur Respir J. 2018;51(4):1702465. doi: 10.1183/13993003.02465-2017

21. Sansone GR, Frengley JD, Vecchione JJ, Manogaram MG, Kaner RJ. Relationship of durantion of ventilator support to successful weaning and other clinical outcomes in 437 prolonged mechanical ventilation patients. J Intensive Care Med. 2017;32(4):283-291. doi: 10.1177/0885066615626897

22. Souza LC, Guimarães FS, Lugon JR. Evaluation of a new index of mechanical ventilation weaning. J Intensive Care Med. 2015;30(1):37-43. doi: 10.1177/0885066613483265

23. Cerqueira FA, Brandão MAG, Mattos VZ, Castellões TMFW. Investigação da temporalidade das características definidoras do diagnóstico de resposta disfuncional ao desmame ventilatório. Esc Anna Nery Rev Enferm. 2012;16(3):545-52. doi: 10.1590/S1414-81452012000300017

24. Maggiore SM, Battilana M, Serano L, Petrini F. Ventilatory support after extubation in critically ill patients. Lancet Respir Med. 2018;6(12):948-62. doi: 10.1016/S2213-2600(18)30375-8

25. Silva LDA, Almeida MMMF, Quaresma MO, Castro T, Santos MA, Chiavegato LD. Success or failure predictive indexes of extubation in renal transplants patients under mechanical ventilation - pilot study. Man Ther Posturology Rehabil J. 2016;14:337. doi: 10.17784/mtprehabJournal.2016.14.337

\section{Autor correspondente}

Marcos Antônio Gomes Brandão

E-mail: marcosbrandao@ufrj.br

Endereço: Departamento de Enfermagem Fundamental - Escola de Enfermagem Anna Nery. R. Afonso Cavalcanti, 275, Cidade Nova - Centro, Rio de Janeiro, RJ, Brasil.

CEP: 20211-130.

\section{Contribuições de Autoria}

1 - Priscila Carvalho de Souza

Concepção e planejamento do projeto de pesquisa, obtenção e análise dos dados, redação.

2 - Ingrid Régia Lopes Jerônimo 
17 | Souza PC, Jerônimo IR, Vianna CA, Benevides AB, Santana RF, Brandão MAG

Concepção e planejamento do projeto de pesquisa, obtenção dos dados, redação.

\section{3 - Carla de Azevedo Vianna}

Interpretação dos dados, redação e revisão crítica.

\section{4 - Andrea Barroso Benevides}

Interpretação dos dados e redação.

\section{5 - Rosimere Ferreira Santana}

Redação e revisão crítica.

\section{6 - Marcos Antônio Gomes Brandão}

Concepção e planejamento do projeto de pesquisa, análise e interpretação dos dados, redação e revisão crítica.

\section{Como citar este artigo}

Souza PC, Jerônimo IR, Vianna CA, Benevides AB, Santana RF, Brandão MAG. Teste de respiração espontânea como preditor da Resposta disfuncional ao desmame ventilatório em terapia intensiva. Rev. Enferm. UFSM. 2020 [Acesso em: Anos Mês Dia]; vol.10 e67: 1-17. DOI:https://doi.org/10.5902/2179769242427 